\title{
Goce, Deseo y Angustia Consideraciones a partir de la Adicción
}

\section{Miren Aranguren ${ }^{1}$}

A través del presente trabajo se analizan algunas de las diferencias que se dan entre el goce y el deseo, basadas sobre todo en la vinculación: relación con el Otro y con el mundo, indispensable para el desarrollo del ser en todas sus etapas y piedra angular en la psicoterapia relacional. Se habla acerca de la necesidad humana de estar en conexión con los propios deseos como medio para establecer y dirigir el proyecto vital. Se expone cómo el goce distancia al sujeto del deseo y con ello lo priva de las funciones adaptativas que le son propias y que pueden llevarnos a una existencia responsable y en definitiva, autónoma. Finalmente se mencionan algunas características de la psicoterapia relacional que se sitúa como una posibilidad psicoterapéutica particularmente interesante para el tratamiento de estos pacientes por poner el foco en la vinculación. Trasversalmente se habla de la toxicomanía, que ilustra lo comentado dado que la comprensión dinámica de la adicción está estrechamente relacionada con el goce del individuo.

Palabras clave: goce, deseo, vinculación, angustia, psicoterapia relacional, psicología existencial.

Through this work there are analyzed the differences between enjoyment and desire, mostly based on human bonding: relationship with the Other and the world, essential for the being development in all of the stages and cornerstone in the relational pshychotherapy. It talks about human necessity of being in conection with the own desires as a resource to stablish and manage the life project. It exposed how enjoyment separates subject from his desires and privates him of adaptative functions wich are own and can drive us to a responsible and ultimately, self-sufficient existence. Finally it mentions some characteristics of relational psychoterapy wich is situated as a particulary interesting psychotherapeutic possibility for the treatment of these patients because its focus on human bonding. It talks about toxicomany transversaly, due to the close relation between addictions dynamic comprehension and the individuals enjoyment.

Key Words: enjoyment (jouissance), desire, angish, human bonding, relational psychotherapy, existential psychology.

English Title: Enjoyment, desire and angish. Drug addiction considerations.

\section{Cita bibliográfica / Reference citation:}

Aranguren, M. (2021). Goce, Deseo y Angustia. Consideraciones a partir de la adicción. Clínica e Investigación Relacional, 15 (1): 259-271. [ISSN 1988-2939] [Recuperado de www.ceir.info ] DOI: 10.21110/19882939.2021.150113

\footnotetext{
${ }^{1}$ Miren Aranguren Lasheras (Pamplona, 1991). Graduada en Psicología (UPV-EHU), continúa su formación en Logoterapia y Análisis Existencial respondiendo a su interés por comprender las condiciones que compartimos y que son inherentes a la existencia individual. Su primer contacto con la Psicoterapia Relacional lo realiza en Talitha Qum (Pamplona). Empieza su trayectoria laboral realizando grupos psicoeducativos y apoyo individual en ANASAPS (Asociación Navarra para la Salud Psíquica) con personas con enfermedad mental y familiares, así como en el centro penitenciario de Pamplona. Actualmente trabaja en Avanvida como educadora en asistencia residencial con personas con enfermedad mental y es alumna en Ágora relacional.
} 


\section{Introducción}

Este trabajo nace de una reflexión acerca de la idea de goce, concepto que se ha relacionado con diferentes formas psicopatológicas como la adicción, a la que iré haciendo breves referencias para ilustrar el lugar que éste ocupa en la vida del sujeto. En su comprensión dinámica, el consumo de la droga lleva al individuo al goce. De la misma manera pienso que es importante aproximarnos a la idea de goce, súmmum de placer, como algo que forma parte de nuestras vidas al igual que lo hace el sufrimiento; a mi entender, casi podríamos considerarlos polos opuestos formando parte del mismo continuum.

Algunas de las preguntas que me planteo son: ¿Hacia dónde nos lleva el estado de goce? ¿Qué lugar ocupa en la vida del ser humano? ¿Cuándo es una expresión psicopatológica? ¿Es siempre el goce la satisfacción de un deseo? ¿Cómo se relacionan ambos conceptos y qué diferencias implican? Y finalmente, ¿qué consideraciones podríamos tener en cuenta en la psicoterapia con estos pacientes?

Más allá de las raíces etiológicas por las que el sujeto tiende o bien al goce, o bien al deseo, baso este trabajo en la idea de que la diferencia fundamental entre goce y deseo radica en la relación con el Otro, o el objeto. En esta relación que se da a partir del momento del nacimiento en los primeros vínculos con los cuidadores, en concreto con la madre, también empieza a gestarse la relación que el individuo va a establecer con el mundo; relación que más bien, uno mismo va a crear a partir de las estructuras mentales desarrolladas a través de la relación. La integración de este vínculo primario que para el sujeto representa lo que el mundo es y que con el paso de los años será aumentado con la vivencia de experiencias y relación con el entorno, será por lo tanto, parte importante de su percepción del mundo, de la forma en que le otorga significados y se vincula con éste.

Como a continuación desarrollo, en la expresión psicopatológica del goce no hay vinculación con el otro, si bien esta ausencia no deja de ser una forma de vinculación específica per se, dado que uno no puede ser sin un otro. El objeto, lo de fuera, siempre está ahí. Así es que me interesa pensar acerca del lugar que ocupa la vinculación a la hora de definir las relaciones que establecemos con el goce y el deseo, siendo clave para comprender algunos de los elementos que considero que van a terminar perfilando relaciones más o menos sanas con los conceptos que dan nombre a este trabajo.

En esta forma de ausencia de vinculación con lo externo, la satisfacción del goce, el goce por el goce, parece ocupar el mayor protagonismo en la vida del sujeto y lo aleja así del desarrollo de su autonomía. Me interesa comenzar esta reflexión en la forma en que el sujeto 
enganchado al goce se relaciona con su mundo y la función que el estado al que llega a través de él cumple en esta forma de relación.

\section{El goce}

En contraste con el deseo que aparece siempre en relación a un objeto y su satisfacción apunta hacia afuera, el goce siempre va destinado a uno mismo, el sujeto vuelca y enfoca la energía hacia sí mismo. Es en este sentido que se le atribuye la ausencia de vinculación. Uno goza a través de su propio cuerpo y mente del estado al que le lleva el acto con el cual goza. A partir de esta breve introducción podemos entender por qué cuando se ha hablado acerca del goce se ha hecho unido a conceptos como el narcisismo o el autoerotismo, que se le han atribuido como características esenciales.

En el caso de la toxicomanía la sustancia ocupa el lugar del objeto para enfocar la energía en uno mismo, con lo que el Otro queda desplazado o sustituido. Esto se puede observar en el deterioro que a menudo sufren las relaciones interpersonales y la actividad del adicto como el trabajo y algunas aficiones de las que generalmente disfrutaba antes de iniciar el consumo. Así, la sustancia acaba reemplazando el lugar del objeto más allá de la cuestión del Otro, más allá del deseo y por encima de todo. La satisfacción del goce (el consumo y la búsqueda de la sustancia en este caso) se vuelve un imperativo y eje central en la vida de la persona. Como sabemos existen diversos factores, biológicos, psicológicos y sociales que pueden influir en la vulnerabilidad y desarrollo de la adicción. Siguiendo la línea de este trabajo cabe preguntarnos hasta qué punto es el estado de goce un elemento relevante que engancha al sujeto en la búsqueda de más goce y con ello a la conducta que lo produce que acaba volviéndose adictiva. Este planteamiento nos llama a la exploración de cómo éste es vivido, a indagar en qué es lo que encontramos en el estado de goce y hacia dónde lleva al sujeto en la ausencia de este otro.

Siguiendo la teoría freudiana, Chasseguet-Smirgel (1975) considera que a través del goce el sujeto anhela volver a un estado propio del narcisismo primario donde se efectúa una refusión con el Otro materno. Esta fusión implica la vuelta a la ilusión de completud propia de las etapas preedípicas, a la omnipotencia infantil donde no existen los límites que la realidad impone ni tampoco la diferenciación entre el yo y los otros. El mundo es uno mismo. Es en el narcisismo primario de donde se deriva el ideal del yo. En este estado simbiótico con la madre protectora aunque el sujeto pierde su individuación se vive a sí mismo completo y perfecto. Es decir, se vive en el acto puro y aislado del goce; sin temporalidad, sin posibilidad de pasar a la reflexión. Esta experiencia generalmente inconsciente podría observarse en los efectos de la intoxicación por algunas drogas que provocan la emergencia de sentimientos 
de omnipotencia, aumento de la percepción de las capacidades o experiencias subjetivas de expansión de la mente y de sentir una especie de conexión trascendental con el universo. Algunos pacientes refieren ser capaces de realizar ciertos actos bajo los efectos de las drogas que sin ellas no lo serían. De esta manera la brecha entre el Yo y el Yo ideal se siente reducirse, abierta para siempre tras la separación de la madre que da fin al narcisismo primario.

Otros autores como Khantzian, Dodes, Johnson, Kernberg y Kalina, aportan ideas acerca de las causas psicológicas que podrían intervenir en el desarrollo de la adicción. Todos ellos abordan el tema profundizando en lo que el sujeto podría estar buscando compensar a través de la experiencia del consumo. Sus consideraciones me ayudaron a ampliar la visión en mi forma de entender qué busca el sujeto mediante el goce y a darle un lugar en nuestra existencia. Las menciono brevemente:

Para Khantzian (1995) debido a una relación deficitaria con los cuidadores primarios, el adicto no ha podido internalizar la capacidad de autorregulación de los afectos y autocuidado. En consecuencia encuentra en las drogas una forma de recurso que compensa estas dificultades. Según Dodes $(1990,1996)$, existe una vulnerabilidad narcisista a sentirse abrumado por experiencias de impotencia e indefensión. Mediante la conducta adictiva la persona puede restaurar cierto sentimiento de poder en oposición a esta vivencia. Johnson (1993) entiende la conducta adictiva como una forma de lidiar con la soledad debido a que las etapas preedípicas no han podido ser resueltas y por lo tanto no se ha podido internalizar la permanencia del objeto. El individuo no puede tolerar la soledad ni satisfacer sus necesidades de dependencia de forma adecuada en una relación. Kernberg (1975) también habla de la conducta adictiva como medio de evitar la experiencia interna de abandono. $Y$ por último, Kalina (1988) encuentra en las causas de la adicción una dificultad para manejar los límites: límites entre el yo y los otros o nuestra condición de finitud por ejemplo. Con la negación de los mismos el sujeto niega una realidad que como dice este autor, no se ajusta a nuestros deseos e intereses.

En mi interés por profundizar y extender el concepto de goce más allá de la adicción trato de buscar un espacio común donde puedan encontrarse las diferentes ideas que aportan los autores. Me pregunto si el anhelo por volver al narcisismo primario a través del goce podría suponer una forma de manejar estas dificultades $y$, es en este punto donde podemos empezar a aumentar la vista del goce para situarlo más allá del lugar que se le ha dado en la psicopatología. Las dificultades que se mencionan probablemente nos resulten familiares, intrínsecamente humanas, al igual que la búsqueda de goce en nuestra inclinación hacia sensaciones placenteras y el anhelo de vivir una vida plena (con el significado que cada uno 
de nosotros le quiera dar). Sentimientos de incertidumbre, competencia o indefensión, la soledad y los límites, son elementos que generalmente subyacen a una gran variedad de experiencias emocionales que emergen en el vínculo que establecemos con otras personas; presentes en la amplia gama de intercambios relacionales. La regulación de las emociones y el autocuidado forman parte de nuestros recursos más importantes, primarios y sofisticados al mismo tiempo, que se desarrollan y actualizan constantemente en el transcurso del tiempo. Así la manera en que cada individuo lidia con estas dificultades va a constituir un aprendizaje esencial en el desarrollo del ser determinando una forma de estar en el mundo que terminará perfilando su autonomía. Al contrario, las dificultades en la integración de estas capacidades a menudo se traducen en diferentes formas desadaptativas de acción e interacción.

\section{La angustia}

Mi propuesta para la comprensión del goce, muy influida por la psicología existencial, es acogerlo como una forma de evadir la angustia emergente ante la percepción, ya sea consciente, inconsciente o simplemente intuitiva, de estas condiciones inevitables que al fin y al cabo, nos acercan a la muerte, al aislamiento de sentirse separado del resto o a la responsabilidad de verse uno mismo como único autor de la propia vida. En oposición a la omnipotencia infantil, uno se percibe a sí mismo en un mundo que no podrá conocer ni comprender desde una certeza absoluta, sino desde su conocimiento biográfico, su subjetividad, que configura la realidad en que vive y se vive a sí mismo, condicionada por sus primeras experiencias y que no puede ser concebida de otra manera que no sea limitada. Si bien a menudo este plano no pasa por una elaboración consciente, se mantiene latente en nuestras vivencias cotidianas amenazando con aparecer en determinados momentos. Comprender e integrar las experiencias por las que uno sufre forma parte del camino hacia la autonomía; uno se ve obligado a lidiar con la angustia que empuja para salir en esta ausencia de verdad movilizando todos los recursos de los que disponemos, a la que el sujeto enganchado al goce parece resistirse en su renuncia a lo que está fuera de él. Obviamente no desde una elección consciente sino desde la dificultad de vinculación, que de alguna forma parece protegerlo de esta falta de suelo firme. Precisamente podríamos entender este "suelo" cimentado mediante el soporte que ha sido recibido a través del vínculo primario y el apego, al sostén recibido en las emociones generadoras de ansiedad en el niño, atribuyéndole al apego seguro mayor firmeza y capacidad de vinculación en contraste con los apegos inseguros o desorganizados que a menudo presentan algunos pacientes. En definitiva, debemos ser capaces de tolerar y sostener cierta dosis de angustia para situarnos 
en el mundo y desarrollar una existencia de manera genuina, únicamente posible en interacción con lo que está fuera que nos devuelve una imagen del sí mismo, revela nuestros recursos y nos permite conocer y desarrollar lo que cada uno es.

Cuando existe un déficit en las primeras relaciones con los cuidadores surgen dificultades tanto en la vinculación como en la mentalización que como sabemos, nos permite entender el comportamiento propio y ajeno en términos de estados mentales, regular las emociones y el comportamiento interpersonal. La capacidad de mentalizar actúa en la forma en que los contenidos de nuestra mente son vividos y se derivan de ella funciones mentales como la capacidad para registrar e identificar los propios deseos o la capacidad reflexiva. Participa en la constitución de las relaciones que establecemos tanto con los demás como con nosotros mismos. Se comentaba antes que el goce del adicto queda fuera de toda representación simbólica del pensamiento y esto lo hace difícil de cuestionar, no pasa por estados reflexivos, no llega a mentalizarse. La adicción podría volverse una forma de manejar la angustia cuando no se encuentran medios para integrarla y resulta intolerable. Quizás debido a un sentimiento de indefensión ante una realidad que se percibe difícil de abarcar. De este modo el sentimiento de angustia podría verse sustituido por la ilusión de sentirse completo, de sentir el yo navegando en un estado sin límites que lo enfrenten a las demandas de la existencia individual, de los otros y de la realidad. La adicción en definitiva, podría tornarse un medio por el cual el sujeto intenta reafirmar su posición en el mundo que no puede verse reafirmada por otros medios si enfrentar esta realidad implica enfrentar la angustia subyacente, constituyendo inconscientemente un riesgo mucho mayor.

Puede ocurrir entonces que la búsqueda del goce y su satisfacción llegue a sustituir la relación que el sujeto tiene con el mundo, desalineado y alejado ya del deseo que resulta necesario como motor para la vinculación y el desarrollo del ser. Moreno (2006) refiriéndose a la drogadicción afirma que la droga llega a sustituir el deseo. Podríamos decir en este caso que el deseo ha sido sustituido por el goce. El yo no puede conducirse a sí mismo sin deseo y debido a la fuerza con la que el goce se impone, como si el sujeto fuera secuestrado, difícilmente podrá guiarse a través de él. De esta forma el imperativo de goce disuelve toda vinculación posible con el deseo inicial. Lo vemos por ejemplo cuando el consumidor en un comienzo busca sensaciones placenteras en la sustancia que aunque en un principio podrían responder a un deseo, con el tiempo y la repetición acaba convirtiéndose o bien en un hábito que no se cuestiona, o bien en necesidad para el alivio de los síntomas de la abstinencia. El sujeto olvida o pierde aquellas posibilidades que podían beneficiarle del goce sin violencia, el goce como la satisfacción de un deseo. Aquí encontramos un atributo de autodestrucción en el goce. La satisfacción del goce por el goce mismo en la medida en que aísla al sujeto, también lo lleva a la destrucción. Así, se ve a menudo el aumento progresivo del consumo de 
drogas que invade cada vez más esferas de la vida del sujeto, la culpa, el vaivén entre estados maníacos y depresivos y los perjuicios físicos y psicológicos que pocas veces acaba siendo compatible con el desarrollo del potencial individual y una vida autónoma.

\section{El deseo}

La capacidad de desear al igual que el mundo emocional, constituye una de nuestras funciones adaptativas para desenvolvernos en el mundo. La emergencia de un deseo concreto percibido se traduce en lenguaje y está sujeto a unos límites que lo definen, por lo que permite en cierta medida una tarea de elaboración que propone una dirección. A la percepción del deseo le sigue una representación que puede posibilitar el paso a la reflexión. En relación con lo que afirmaba en un principio, en contraste con el goce donde la energía se vuelve al sí mismo, el deseo siempre se dirige al Otro, a lo externo (aunque en su comprensión psicoanalítica tenga su origen en experiencias infantiles que ahora son inconscientes). Ante lo infecundo del goce sin vinculación, en el deseo germinan la motivación y la acción de ir hacia alguien o hacia algo, ofrece posibilidades, lo que lo convierte también en un medio de autorrealización. Es una forma de trascender el propio yo, y su actuación nutre y enriquece la experiencia. Esto me recuerda el concepto de autotrascendencia de Viktor Frankl, con lo que relaciono la idea de deseo que expongo. Para Frankl (2002) la autorrealización no puede ser una meta en sí misma (que también estaría relacionada con el sí mismo y bien podría considerarse un fin narcisista) sino que es producto de la autotrascendencia. Frankl lo explica de esta manera: "El hombre apunta por encima de sí mismo hacia algo que no es él mismo, hacia algo o alguien, hacia un sentido cuya plenitud hay que lograr o hacia un semejante con quien uno se encuentra". Implica por tanto una forma de vinculación con lo que está fuera. Coincido con este autor en que el apuntar hacia algo o hacia alguien se nos presenta como un indicador de salud mental. El deseo guarda el potencial de encontrar sentidos para nuestra existencia. Así es que la relación establecida con el deseo también podría participar en la expresión de otros trastornos psicológicos, como en el caso del obsesivo, que tiene dificultades para conectar con su deseo puesto que no sabe lo que desea, o el depresivo, quien siente que su deseo ha desaparecido.

Decía que cuando el deseo es percibido e integrado en la experiencia propia otorga una dirección; uno tiene que contemplar sus posibilidades y elegir entre ellas para la satisfacción del mismo. Su satisfacción generalmente, tiende a un sentido que cumple en alguna medida con una coherencia dentro de la propia historia y proyecto vital, donde el yo está mediando; un yo que se encuentra limitado a una personalidad y contexto determinado que decidirá cómo responder ante éste. Tal y como hemos visto hasta ahora, el goce se impone como 
deseo violento y tira del sujeto hasta sumergirlo en un estado carente de límites, donde el yo acaba desapareciendo como instancia mediadora entre las diferentes fuerzas. Desde ahí uno no puede realizar la labor de elegir y tomar una decisión, se ha abandonado a sí mismo. Al contrario de lo que sucede cuando uno puede hacerse cargo de su deseo, el goce distancia al yo de su verdadero ser y de los elementos existenciales que le son propios como medio de crecimiento y autodescubrimiento. Al desaparecer los límites se pierde la conciencia del yo y con ello toda posibilidad de generar una vida auténtica. El estado al que lo lleva acaso pueda hacerlo sentir fundido con la totalidad de la existencia. Sin embargo, lo acerca a la nada; no hay nada más allá del acto puro del goce. Creo que una relación satisfactoria con el deseo, basada en su comprensión, discriminación y consecuente creatividad necesaria para convertir el impulso del deseo en acción, es lo que da en gran parte sentido a la propia vida; como dice Frankl apunta hacia un sentido. Para Yalom (2010) el proceso de convertir el deseo en acción requiere un compromiso, así es que según él, el acto responsable empieza con un deseo. Uno asume entonces la condición de libertad al elegir entre sus posibilidades, se compromete con una dirección y modela su existencia concreta en el mundo; este es el concepto de responsabilidad al que se hace alusión.

De esta manera al deseo le sigue la libertad de elegir y la voluntad de llevarlo o no a cabo, mientras que la autoridad incuestionable del goce vuelve a ambas más débiles. El craving sería un ejemplo ilustrativo en la toxicomanía. Todo esto nos va dando claridad en la relación entre el goce y las patologías que implican actos compulsivos que a menudo el sujeto no quiere realizar pero que se ve obligado a ello. Lo mismo sucede con el concepto de responsabilidad: mientras que de alguna manera uno puede responsabilizarse de su deseo (en cuanto lo reconoce como suyo) y el acto que lleva a cabo tras su experimentación, en el goce su satisfacción se vuelve un imperativo por lo que difícilmente el sujeto puede hacerse cargo de este impulso, cuestionarlo o tomar una decisión. Al contrario de la vivencia de expansión que se siente vivir a través del goce, el individuo restringe sin darse cuenta todas sus posibilidades de responsabilizarse de su propia existencia. Tampoco podrá desde ese estado elaborar aquello que no le ha permitido desarrollar estrategias adaptativas de vinculación con el otro, dificultándole la consecución de su autonomía. Pienso que en el proceso de compromiso al que alude Yalom, llamado por el deseo y que lleva al individuo a responsabilizarse de su existencia y posición en el mundo, se encuentran las condiciones necesarias para generar experiencias mediante las cuales uno puede enfrentar la angustia subyacente. De esta forma uno está inmerso en la búsqueda de los significados de su existencia que podrán protegerlo ante ella.

El deseo se hace un hueco en el espacio y tiempo del individuo, que, aunque inexorablemente influido por experiencias pasadas, conecta con el presente y el futuro. $A$ 
diferencia de la fantasía de completud atemporal a la que el sujeto vuelve en el goce, en la expresión del deseo encontramos la totalidad del tiempo, lo que el sujeto es, su historia y su proyecto, que en dirección a los otros y al mundo nos orienta hacia la completud a la que a mi entender podemos aspirar los seres humanos. No cabe duda de que el deseo necesita de una representación para existir, un impulso de ir hacia alguien o hacia algo que se enfoca en el futuro y constituye su presente. Gracias a la integridad del deseo, uno puede crear y comprometerse con un proyecto de vida con el que pueda sentirse identificado, en contraste con la mirada que el goce dirige al pasado en el anhelo fusional que nunca más será alcanzado y que lo privará de enfrentar una existencia individualizada.

Considero por tanto que el deseo es potencialmente el recurso movilizador de aquello que define lo esencial de la existencia humana: la libertad, la responsabilidad, el sentido, la voluntad. El deseo tiende a proyectarse a lo externo y nos mueve a la asunción de todas estas condiciones que van a dar forma a la existencia concreta y personal del individuo en la medida en que las lleva al plano de la posibilidad y permite que se traduzcan en acto. Es en este proceso donde uno puede sostenerse y asomarse ante el precipicio de la angustia que nos amenaza con aquello que tememos desde planos más oscuros: la muerte, el sinsentido, la soledad, la incertidumbre, el tedio.

\section{Deseo y vinculación en la psicoterapia relacional}

La psicoterapia relacional se sitúa como una opción de tratamiento que, dada la importancia que atribuye a la relación que se establece entre terapeuta y paciente, donde reside gran parte de su potencial terapéutico, puede resultar muy adecuada con estos pacientes cuyas dificultades surgen en parte por su historia en la vinculación con los otros. De hecho, parecen haber encontrado en la sustitución de este otro una forma de sostenerse. La idea es que el paciente pueda volver a vincularse y que esta vinculación cumpla con un sentido para él. Como ya he mencionado anteriormente, es probable que debido a los déficits experimentados en los primeros vínculos no se haya podido desarrollar una forma de relación en la que sentirse seguro y confiado. Puede que la ruptura con los otros constituya un intento de autoprotección, pues considero que a través de la droga el sujeto trata de defenderse de algo. Quizás la vivencia del goce suponga un riesgo menor que la exposición al mundo de fuera y a la interacción con otras personas, donde se teme que las primeras experiencias de relación sean confirmadas y lo lleven a revivir la ansiedad y el dolor sentido de niño, que en algún lugar de su mente se recuerda vulnerable e indefenso ante tal realidad.

Se trata entonces, de crear primero un espacio seguro donde el paciente pueda sentir la confianza necesaria para explorar después, a lo largo del proceso, algunos elementos de su 
existencia que previamente no había podido contemplar. Esto pasa por la toma de conciencia y el análisis de los primeros vínculos, que serán inevitablemente revividos a lo largo del transcurso de la psicoterapia y que gracias a la implicación del paciente y a la atenta observación del terapeuta, podrán ser percibidos, mencionados y reflexionados. A través de la comprensión del poder constituyente de los primeros vínculos, que han configurado una manera de "filtrar" y de dar un significado a la realidad que el sujeto vive en su vida y sus relaciones, podrá irse ampliando este filtro y con ello la persona podrá asomarse a algunas de las circunstancias de su vida que hasta ese momento no podía ver. El contexto terapéutico ofrece al paciente la posibilidad de ser creador, junto con el terapeuta, de una nueva forma de vincularse que permita reestructurar algunos de los mecanismos instaurados a través de los anteriores vínculos que no le han permitido desarrollar formas satisfactorias de relación con los otros y que posiblemente haya influido en el desarrollo de una adicción en un intento de adaptarse a las exigencias percibidas. El terapeuta deberá velar por el establecimiento de este vínculo seguro en su forma de relacionarse con el paciente, reconocerlo y validarlo tal y como es, de manera que éste pueda verse a sí mismo, en su historia y sus afectos; en su manera de crear y percibir el mundo en el que se vive.

Para Olievenstein (1983) "el rol del terapeuta es aquel que conduce poco a poco al deseo del paciente de construir una identidad diferente de aquella del drogadicto". Y es cierto que una vez la adicción invade la vida del sujeto, desconectándolo de su deseo y libertad y siendo desconocedor de su potencial en cuanto a lo que tiene para aportar al mundo, le es difícil identificarse con una identidad que no sea la de adicto. Esto no le permite profundizar en su yo y conocerse, ni tampoco dirigir libremente su vida, componentes importantes para la definición y realización del yo.

Según Joan Coderch (2012), los resultados del análisis se miden en gran parte por el grado en que el paciente ha internalizado una actitud de continuada introspección y autoanálisis. De esto trata pues, en definitiva, la labor del psicoterapeuta. Ha de promover en el transcurso de la terapia y al ritmo del paciente (pero siempre sin perder de vista este objetivo) un espacio que le permita explorar su mundo interno e interpersonal, de manera que con el tiempo se convierta en una capacidad personal y así pueda elegir su forma de posicionarse ante lo conocido a lo largo de este proceso y ante las experiencias futuras. Esta actitud se corresponde en gran parte con la capacidad de reflexionar y mentalizar, donde suelen encontrar dificultades los pacientes con patologías adictivas y por lo que se vuelve importante en el tratamiento.

Para ello es imprescindible traer la subjetividad del paciente al espacio psicoterapéutico. Otra vez en palabras de Coderch, "el analista devuelve al paciente lo que ha recibido para 
que éste lo reconozca como suyo y lo vaya integrando para reconocer su propia subjetividad". En la medida en que el paciente vaya familiarizándose con la emergencia de su propia subjetividad, de su manera de ser en el mundo, más fácilmente podrá conocer su manera de sentir, su deseo y su voluntad, y podrá enfocarse hacia experiencias con sentido atravesando la violencia del goce en su búsqueda de realizarse a sí mismo. No podemos olvidar la importancia de que el paciente pueda conectar con sus deseos. En el anterior apartado se le atribuía la característica de motor para la vinculación y el desarrollo del ser, por lo que también será el elemento indispensable en el proceso psicoterapéutico que definirá la orientación hacia el cambio deseado y gracias a lo que podrá cultivar la motivación necesaria en el transcurso de la terapia.

Por último me gustaría añadir otra consideración a la que personalmente atribuyo gran valor en el trabajo psicoterapéutico. Se trata de la mirada con la que vemos a los pacientes. Creo que siempre y especialmente en los casos donde parte de la confusión del paciente hace referencia a su identidad, resulta esencial poder ver más allá de su experiencia concreta, de lo que lo aqueja, de sus dificultades. La capacidad de percibir el potencial que aún está por desarrollar en la vida del paciente y mostrar la confianza y convicción interna de que así podrá ser, forma parte de esa comunicación no hablada que también se da en la relación. De alguna manera cuando ésta es genuina por parte del terapeuta el paciente puede percibirla. Coderch menciona algo parecido de la siguiente manera: "me parece de suma importancia que los pacientes capten que el terapeuta tiene una imagen de ellos en su mente en un nivel de desarrollo superior al que se hallan. Imagen con la que ellos, en los casos favorables, van identificándose". Pienso que es oportuno mencionarlo tras todo lo comentado hasta aquí pues en el mejor de los casos, esta identificación del paciente con la imagen que el terapeuta crea en su mente acerca de él podría alentarlo a la construcción de su identidad y su proyecto.

Este último elemento nos revela hasta dónde llega el alcance de la vinculación con los otros. La imagen que el otro tiene de uno mismo, o lo que este último intuye que el otro percibe de él, también contribuye a la conciencia de la idea que cada uno tiene de sí mismo, en la medida en que nos identificamos o no con esta imagen que de diferentes maneras (generalmente no explícitas) nos es devuelta. La vinculación es un medio gracias al cual podemos vernos y establecer la dirección deseada tratando de disminuir, desde la aceptación, la perenne ruptura entre el Yo y el Yo ideal.

\section{Conclusiones}

He hablado del goce siguiendo la teoría freudiana del narcisismo primario, que evoca de forma inconsciente la omnipotencia infantil respondiendo al anhelo fusional, en una especie 
de dejarse fluir a través de una existencia sin límites que va más allá del yo. Al deseo en cambio se le ha atribuido el poder trascendental de generar sentido en la propia vida. Quedan entonces dos preguntas por responder: ¿Dónde se encuentran el goce y el deseo? Cuando gozamos, ¿estamos anulando las cualidades existenciales que nos son propias y dan forma a nuestra vida?

Cuando hablamos del goce desde la psicopatología pensamos en adicciones, comportamientos compulsivos o narcisistas, en autodestrucción. Pero bien podríamos observar el goce de quien forma parte de una orquesta y olvidándose de sí mismo se siente fundido con la música creada en sintonía con los demás músicos. En un deleite gastronómico. La meditación de un budista experimentado o la sensación de catarsis en una representación teatral. La vivencia del amor en sus diversas manifestaciones. Las referencias a la adicción nos pueden ayudar a entender cómo el goce podría obstaculizar el desarrollo de la autonomía paralizando o anulando los recursos que permiten el delineamiento de un proyecto vital, pero no nos hablan del goce en sí mismo sino de la relación que se establece con él, del lugar que termina haciéndose en la vida del adicto. En los últimos ejemplos en cambio podemos percibir al yo que enfocado a su tarea abre paso al goce y permite al sujeto experimentar el placer de su vivencia. Aquí encontramos la diferencia fundamental que va a hacer que el goce forme parte de una expresión patológica o saludable. Es en la labor de mediación e integración del yo que otorga un lugar al goce donde encontramos su máximo exponente, para lo que necesitaremos cierta conexión con el deseo que nos va a alentar a la vinculación y el reconocimiento de lo que no soy yo. La intervención del yo nos permite atribuir significados, representar esta experiencia y mentalizarla a lo largo de diferentes tiempos lo que nos posibilita vivir el goce desde estados más maduros. La vivencia del goce podría generar una especie de curiosidad que alienta al descubrimiento de lo que nos da placer también como rasgo identitario, lo cual nos lleva a descifrar y hacernos cargo de nuestras posibilidades al tiempo que se movilizan nuestros elementos existenciales.

A lo largo de este artículo intento pensar en la función que cumple el goce en la vida de los seres humanos, aunque resulta algo paradójico limitar una experiencia con el lenguaje que precisamente se caracteriza por atravesar los estadios más racionales. Tal vez el estado de goce nos haga olvidar que necesitamos un sentido muy personal para desarrollarnos y disfrutar de nuestra existencia dado que su misma vivencia parece confirmar este sentido y lo lleva hacia la trascendencia. Pareciera una especie de compensación mística en oposición a la vivencia de angustia que de la misma manera es parte de nuestra vida. Aunque antagónicas en nuestro sentir, ambas hacen que nos encontremos con nosotros mismos y nos impulsan a buscar nuestro posicionamiento ante la vida. 


\section{REFERENCIAS}

CHASSEGUET-SMIRGEL, JANINE (1975). El ideal del yo. Ensayo psicoanalítico sobre la "enfermedad de idealidad". Buenos Aires: Amorrortu, 1991.

CODERCH, J. (2012). La práctica de la psicoterapia relacional. El modelo interactivo en el campo del psicoanálisis. $2^{a}$ ed. Corregida. Madrid: Ágora Relacional.

DODES, L.M. (1990). Addiction, helplessness, and narcissistic rage. Psychoanalytic Quarterly 59:398419.

DODES, L.M. (1996). Compulsion and addiction. Journal of the American Psychoanalytic Association 44:815-835.

FRANKL, V. (2002). La voluntad de sentido. $3^{\mathrm{a}}$ ed. Barcelona: Herder pag. 21.

GONZÁLEZ GUERRAS, J. (2008). Psicoanálisis y toxicomanías. Clínica e investigación relacional vol. 2 Pp $146-164$.

JOHNSON, B. (1993). A developmental model of addictions and its relationships to twelve step program of Alcoholics Anonymous. Journal of Substance Abuse Treatment 10:23-34.

KALINA, E. (1988). Adolescencia y drogadicción, (3ª ed.), Buenos Aires: Nueva Visión, 1997.

KERNBERG, O. (1975). Borderline Conditions and Pathological Narcissism. New York: Aronson.

KHANTZIAN, E.J. (1995). Sel-regulation vulnerabilities in substance abusers: Treatment implications. In The Psychology and Treatment of Addictive Behavior, ed. S. Dowling. Madison, CT: International Universities Press, pp 17-42.

MORENO, A. (2006). Cuerpo, toxicomanía y psicoanálisis. Revista Acheronta No. 23.

OLIEVENSTEIN, C. (1983). La drogue ou la vie. Paris: Robert Laffont.

ORTEGA, J. (2008). El goce del adicto: psicoanálisis y toxicomanía. Recuperado de https://psicoanalisisextension.blogspot.com/search?q=goce

YALOM, I. (2010). Psicoterapia existencial. Barcelona: Herder pag. 366.

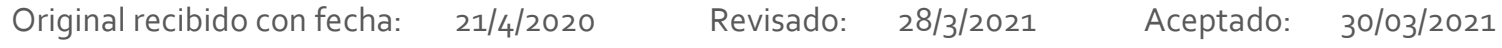

